\title{
Titanium and steel fracture fixation plates with different surface topographies: Influence on infection rate in a rabbit fracture model
}

\author{
W.J. Metsemakers, Tanja Schmid, Stephan Zeiter, Manuela Ernst, Iris Keller, \\ Nicolo Cosmelli, Daniel Arens, T. Fintan Moriarty *, R. Geoff Richards \\ AO Research Institute Davos, Davos, Switzerland
}

A R T I C L E I N F O

Article history:

Accepted 11 January 2016

\section{Keywords:}

Osteosynthesis

Infection

Titanium

Steel

Surface topography

Staphylococcus aureus

\begin{abstract}
A B S T R A C T
Introduction: Implant-related infection is a challenging complication in musculoskeletal trauma surgery. In the present study, we examined the role of implant material and surface topography as influencing factors on the development of infection in an experimental model of plating osteosynthesis in the rabbit. Methods: The implants included in this experimental study were composed of: standard Electropolished Stainless Steel (EPSS), standard titanium (Ti-S), roughened stainless steel (RSS) and surface polished titanium (Ti-P). Construct stability and load-to-failure of Ti-P implants was compared to that of Ti-S implants in a rabbit cadaveric model. In an in vivo study, a rabbit humeral fracture model was used. Each rabbit received one of three Staphylococcus aureus inocula, aimed at determining the infection rate at a low, medium and high dose of bacteria. Outcome measures were quantification of bacteria on the implant and in the surrounding tissues, and determination of the infectious dose 50 (ID ${ }_{50}$ ).

Results: No significant differences were observed between Ti-S and Ti-P regarding stiffness or failure load in the cadaver study. Of the 72 rabbits eventually included in the in vivo study, 50 developed an infection. The $\mathrm{ID}_{50}$ was found to be: EPSS $3.89 \times 10^{3}$ colony forming units (CFU); RSS $8.23 \times 10^{3} \mathrm{CFU}$; Ti-S $5.66 \times 10^{3} \mathrm{CFU}$; Ti-P $3.41 \times 10^{3} \mathrm{CFU}$. Significantly lower bacterial counts were found on the Ti-S implants samples compared with RSS implants $(p<0.001)$ at the high inoculum. Similarly, lower bacterial counts were found in the bone samples of animals in the Ti-S group in comparison with both RSS and EPSS groups, again at the high inoculation dose $(p<0.005)$.

Conclusion: No significant differences were seen in susceptibility to infection when comparing titanium and steel implants with conventional or modified topographies. Ti-P implants, which have previously been shown in preclinical studies to reduce complications associated with tissue adherence, do not affect infection rate in this preclinical fracture model. Therefore, Ti-P implants are not expected to affect the infection rate, or influence implant stability in the clinical situation.
\end{abstract}

(c) 2016 Elsevier Ltd. All rights reserved.

\section{Introduction}

One of the major complications in musculoskeletal trauma surgery is implant-related-infection. These infections are difficult to treat and have a significant socio-economic impact $[1,2]$. In spite of improvements in implant design, improvements in surgical technique, and the routine use of prophylactic antibiotics, implant-related infection remains an ever-present problem [3]. In terms of the role played by the implant itself, various implantspecific design or application features may influence infection

* Corresponding author at: AO Research Institute Davos, AO Foundation, Clavadelerstrasse 8, Davos Platz, CH7270, Switzerland. Tel.: +41 814142397.

E-mail address: fintan.moriarty@aofoundation.org (T.F. Moriarty). susceptibility. Such features include the type of metal (e.g. stainless steel, titanium), the surface topography (e.g. microrough, polished), and the type of plate (e.g. locking compression plate (LCP), dynamic compression plate (DCP)) [4-6].

Stainless steel, titanium and titanium alloys (e.g. titanium-6\% aluminium-7\% niobium, TAN) are the most common materials used in the manufacture of fracture fixation implants $[7,8]$. The difference in infection susceptibility between these metals has been a topic of research for over 20 years [9]. Experimental data in animal studies have indicated that titanium is superior to Electropolished Stainless Steel (EPSS) with regard to infection susceptibility $[4,6,10]$ and this has generally been attributed to superior biocompatibility of titanium and the observation that fibrous capsules tend to form around EPSS implants. A small number of clinical studies [11-14] have seemed to corroborate 
these preclinical findings to a degree, although the effect appears to be marginal and limited to studies with relatively small patient numbers. In an attempt to define the infection susceptibility of common implant materials and topographies in a controlled manner, the infection susceptibility of titanium, TAN and EPSS LCP implants was previously assessed in an experimental setting using a non-fracture rabbit model [15]. However, no statistically significant differences were found between these materials as standard, or when the surfaces of the titanium implants were polished. It is likely that the fracture may be a critical component in the risk of infection since previous studies have shown that fracture stability is of paramount importance with respect to infection prevention and treatment [2].

The aim of this study was to define the role of implant material and surface topography in a preclinical in vivo model incorporating appropriate fracture biomechanics and bone healing. Such information would provide definitive preclinical proof as to whether the material, or surface topography, of fracture fixation devices plays a significant role in infection susceptibility with locking plates.

\section{Materials and methods}

\section{Implant manufacturing}

The LCPs used in this study were commercially available implants for human medicine (52 mm long, straight, 7-hole, $2.0 \mathrm{~mm}$ LCPs, DepuySynthes, New Jersey, USA). In total, four implant variants were included in this study: standard commercially available Electropolished Stainless Steel (EPSS); standard commercially available titanium (Ti-S); surface-roughened Stainless Steel (RSS); and surface polished Titanium (Ti-P) (Fig. 1).
The RSS plates were created from what were originally standard EPSS plates. The plates were roughened with a water jet, operating with an injector diameter of $0.3 \mathrm{~mm}$, water pressure of $3800 \mathrm{bar}$ and a speed of $250 \mathrm{~mm} / \mathrm{min}$. The entire upper and lower surface of the plate was treated, except a small circular margin along the most proximal and distal screw holes, where the plate was held under the waterjet (Fig. 1). The Ti-P plates were created from what were originally Ti-S plates by KKS Ultraschall AG, Switzerland. The polished screws are hereafter named TAN-P, and unpolished standard equivalents TAN-S. All implants were finally intensively rinsed with deionised water and dried. Prior to implantation, all LCPs and screws were rinsed with deionised water and steam autoclaved.

\section{Surface characterisation}

The surface topography of the standard and modified LCPs was quantitatively measured by non-contact, white light profilometry (FRT MicroProf 200 Profilometer, Fries Research \& Technology, Germany). The surface topography was imaged with a Hitachi S4700 field emission scanning electron microscope (SEM) operated in secondary electron (SE) detection mode at an accelerating voltage of $5 \mathrm{kV}$, emission current of $40 \mu \mathrm{A}$ and a working distance of $12 \mathrm{~mm}$.

\section{Mechanical testing}

Construct stability and load until failure of the Ti-P implants was compared to that of the Ti-S implants. Sixteen cadaveric rabbit humeri ( $n=8$ rabbits) were subjected to the same surgical procedure for the Ti-S and Ti-P groups as described for the in
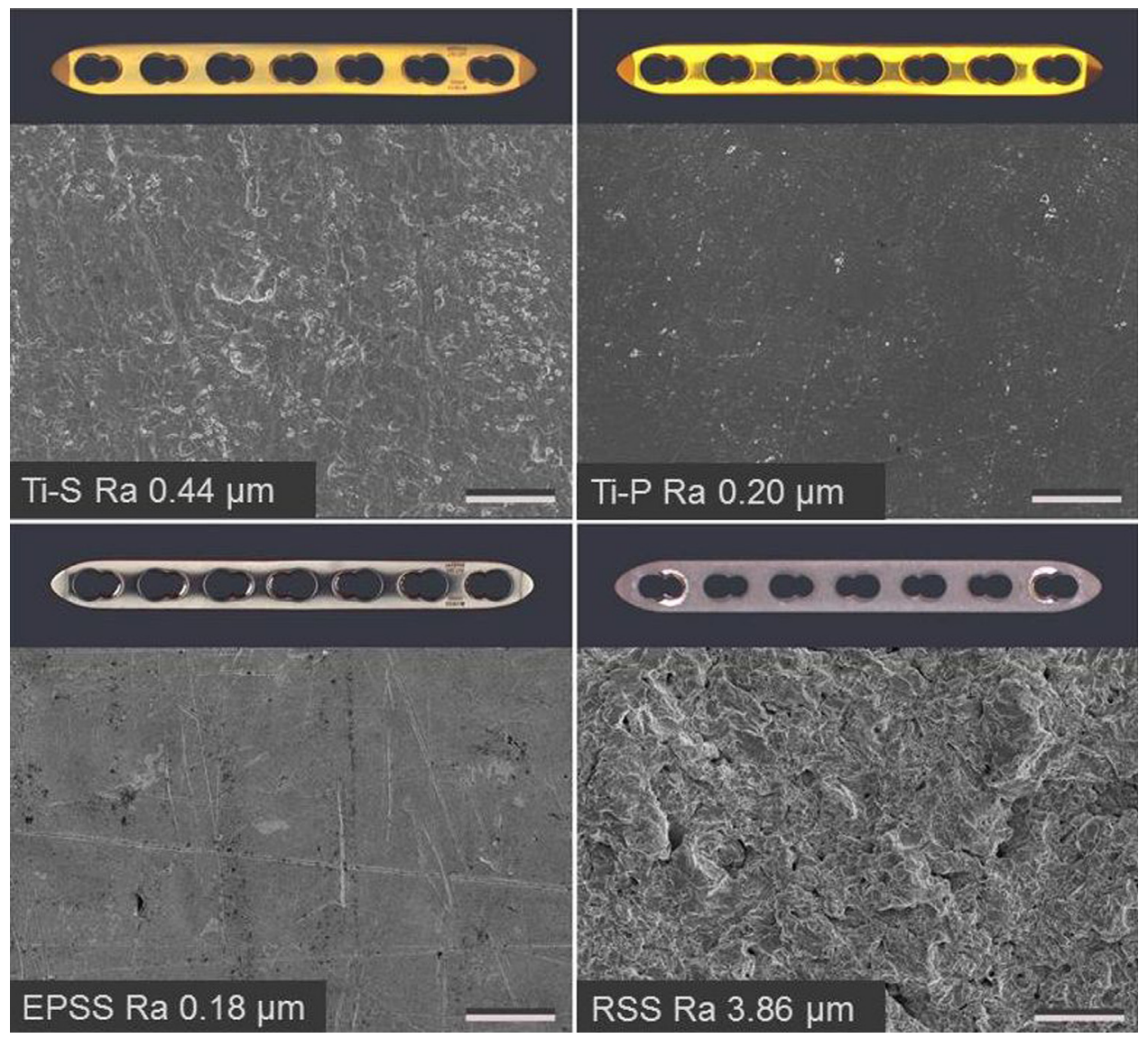

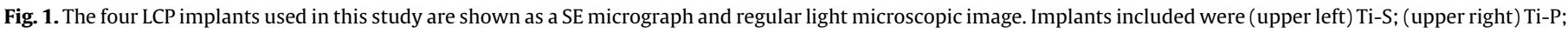
(lower left) EPSS; (lower right) R-SS (scale bar $50 \mu \mathrm{m}$ ). 


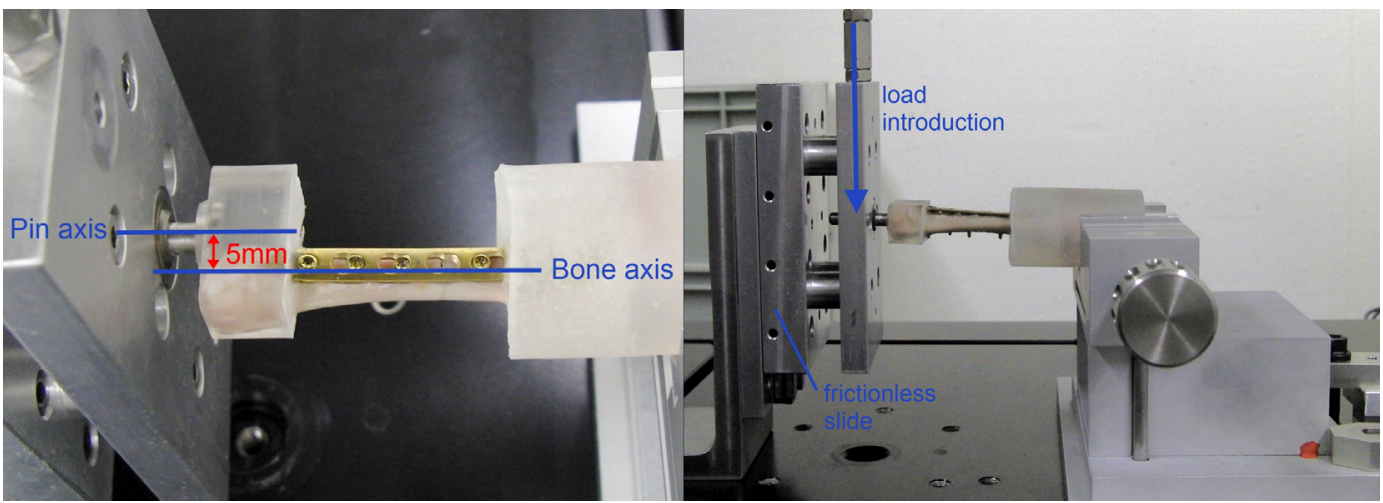

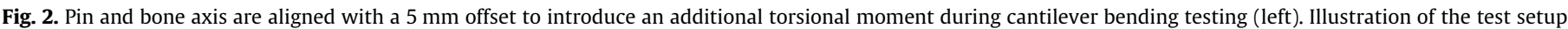

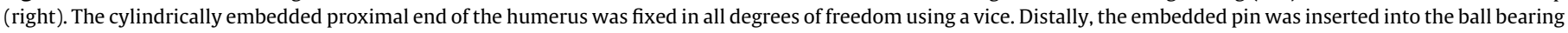
of the vertical slide, which was moved up and down for load introduction during biomechanical testing.

vivo study below. The screw holes were drilled with a $1.8 \mathrm{~mm}$ drill bit for the Ti-S screws. The screw holes for the Ti-P group were drilled with a $1.6 \mathrm{~mm}$ drill bit to ensure the polished screws fully engaged with the bone as electropolishing resulted in a reduction in screw diameter. Both ends of the bone were embedded in polymethylmethacrylate (PMMA) (Troller AG, Fulenbach, Switzerland). The distal end of the humeri was embedded together with a metal pin aligned at a $5 \mathrm{~mm}$ offset with the long bone axis (Fig. 2). Biomechanical testing was performed using a MTS Acumen machine (MTS Systems Corp., Eden Prairie, MN, USA) with a $500 \mathrm{~N}$ load cell coupled to the machine's cross head. For specimen installation, the proximal PMMA cylinder was rigidly clamped in a vice with the plate facing upwards, while the metal pin of the distal end was placed in a ball bearing of a vertical slide with negligible friction (Fig. 2). The slide was attached to the machines crosshead for load introduction, exposing the specimens to cantilever bending forces as well as torsional loading.

In vivo study

\section{Animals, surgical anaesthesia protocol and euthanasia}

The model used was the contaminated plate model of Arens et al. [16], which is an osteotomy model in the rabbit humerus. The in vivo study was approved by the ethical committee of the canton of Grisons in Switzerland (approval number 16/2014; 34F/2014). All procedures were performed in an AAALAC (Association for Assessment and Accreditation of Laboratory Animal Care International) approved facility and according to Swiss animal protection law and regulations. Seventy nine skeletally mature specific pathogen free (SPF) female New Zealand White rabbits (Charles River, Germany) between 24 and 38 weeks of age and a mean body weight of $3.86 \pm 0.49 \mathrm{~kg}$ were finally included in this study.
Anaesthesia and surgery was performed according to the plating procedure previously described [16]. The EPSS and RSS LCPs were fixed using EPSS screws, Ti-S LCPs were fixed with TAN-S screws, Ti-P LCPs were fixed with TAN-P screws. The screw holes were drilled with a $1.8 \mathrm{~mm}$ drill bit for the Ti-S, EPSS and RSS groups, all of which received standard screws. The screw holes for the Ti-P group were drilled with a $1.6 \mathrm{~mm}$ drill bit to ensure the polished screws fully engaged with the bone (as tested in biomechanical test described above). A full mid-diaphyseal osteotomy was created using a $0.44 \mathrm{~mm}$ Gigly saw (RISystem, Switzerland), centred underneath the unused central combi-hole (Fig. 3). Inoculation was performed by pipetting three separate $34 \mu \mathrm{l}$ injections onto the central screw hole overlying the osteotomy and to the adjacent proximal and distal screw holes (total number of bacteria added was $102 \mu \mathrm{l}$ and the number of CFU quantified, as described below).

\section{Clinical observations}

Blood samples were taken preoperatively, 3 days postoperative and weekly thereafter until the end of the observation period for white blood cell (WBC) count (Vet ABC, Scil animal care, Viernheim, Germany). Weight was measured at surgery and weekly thereafter as a criteria for early exclusion. Radiographs of the operated limb were taken in two planes after surgery and once a week thereafter for the rest of the study. Exclusion criteria were set as previously described, and included post-operative fracture of the operated bone. Upon completion of the observation period, all animals were humanely euthanised

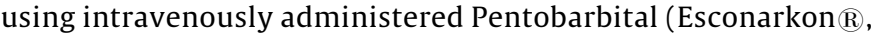
Streuli Pharma AG, Switzerland). A contact radiograph (full thickness) was taken of the operated limb post mortem using

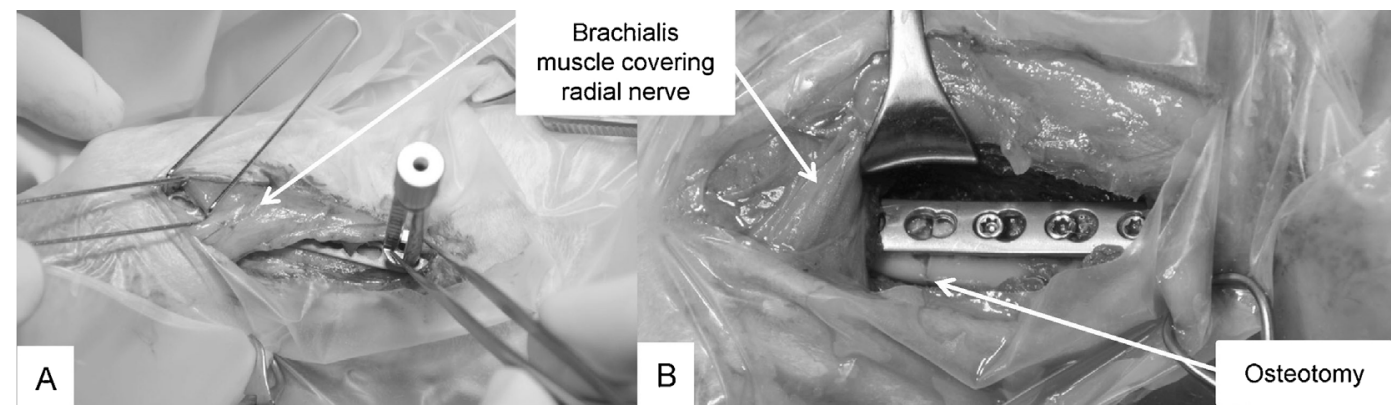

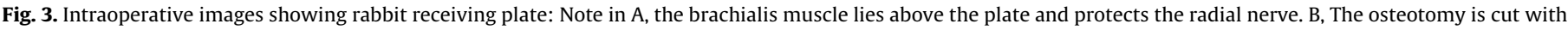
continual protection of both the muscle and nerve [16]. 
high-resolution technical film (D4 Structurix DW ETE, Agfa, Belgium) and a cabinet X-ray system (Faxitron X-Ray Corporation, USA).

\section{Bacteriology}

A clinical Staphylococcus aureus strain (JAR060131), isolated from a patient with an infected hip prosthesis, was used in this study [17]. Each rabbit received one of three inocula, aimed at determining the infection rate at a low $\left(2 \times 10^{3} \mathrm{CFU}\right)$, medium $\left(2 \times 10^{4} \mathrm{CFU}\right)$ or high dose $\left(2 \times 10^{5} \mathrm{CFU}\right)$ of bacteria. The bacterial inocula were individually prepared in Phosphate buffered saline solution (PBS, Sigma-Aldrich, Switzerland) for each surgery. Quantitative culture of each inoculum was performed immediately after preparation to check the accuracy of the prepared inoculum.

Post-mortem bacteriological evaluations were performed on all animals. Quantitative bacterial cultures were performed for the soft tissue, implant and bone in three separate assessments after sonication and/or mechanical homogenisation as described previously [16].

\section{Statistical analysis}

Results are presented as means of each group with standard error of the mean (S.E.M.). Results of biomechanical testing were tested for normal distribution by Shapiro Wilk test, and found to be greater than 0.05 (indicating normal distribution), therefore a student $t$ test was applied for statistical evaluation. A Kruskal-Wallis test was used to compare differences between bacterial burden in different groups at different doses. In all cases, significance was set at ${ }^{*} p<0.05,{ }^{* * *} p<0.001$. Prism software was used for all statistical tests (GraphPad Software Inc., La Jolla, CA). The calculation of the $\mathrm{ID}_{50}$ was based on cumulative infection rates according to the method described by Reed [18].

\section{Results}

\section{Implant surface observations}

The Ti-S LCPs used in this study were found to have an Ra of $0.44 \pm 0.02 \mu \mathrm{m}$ (SD) as measured by white light profilometry. Polishing the Ti LCPs (Ti-P) reduced surface roughness to $0.20 \pm 0.01 \mu \mathrm{m}$. The EPSS LCPS were the smoothest of the tested implants with an Ra of $0.18 \pm 0.02 \mu \mathrm{m}$. The water jet treatment increased the roughness of the steel plates to $3.86 \pm 0.66 \mu \mathrm{m}$, and was thus the roughest of the materials tested. SEM images generally reflected the findings of the white light profilometry, whereby Ti-S showed a rugged surface morphology and Ti-P plates showed a smoother surface. EPSS also had a very smooth surface and RSS displayed the roughest surface (Fig. 1).

\section{Mechanical testing}

Two out of the eight specimens of each group were excluded from the final analysis due to displacement prior to failure. Of the remaining specimens in both groups, only one primary mode of failure was observed. This involved a break in the bone through the most distal screw hole in close proximity to the embedding material. Each group had a single specimen fail due to compression under the plate. No significant difference were observed between the two groups with regards to failure load (Ti-P: $63.81 \pm 8.19 \mathrm{~N}$, TiS: $56.57 \mathrm{~N} \pm 17.26 \mathrm{~N}$ ) and stiffness at 100 cycles (Ti-P: $21 \pm 18 \mathrm{~N} / \mathrm{mm}$, Ti-S: $10 \pm 6 \mathrm{~N} / \mathrm{mm}$ ) or stiffness at 1000 cycles (Ti-P: $22 \pm 15 \mathrm{~N} / \mathrm{mm}$,
Ti-S: $12 \pm 8 \mathrm{~N} / \mathrm{mm}$ ). Importantly, no loosening of the screws could be observed in any of the specimens.

\section{In vivo study}

All 79 animals included in this study survived the surgical procedure, although 3 operated rabbits were euthanised prematurely due to fracture of the humerus close to the osteotomy gap. These fractures occurred within the first 14 postoperative days. One rabbit was excluded in the early postoperative phase due to wound dehiscence, which originated from excessive cleaning of the surgery site by the rabbit. During placement of the TAN-P screws, some screws were found to lack sufficient purchase in the pre-drilled bone, and so these animals $(n=3)$ were excluded and replaced. This observation resulted in the modified predrilling routine for this group, as described above for the surgical procedure and as tested biomechanically. The remainder of the rabbits survived the entire observation period ( 28 days) without any complications resulting in a final group size of 6 per inoculum, per group (total $n=72$ ). WBC counts were elevated in animals ultimately deemed to be infected in comparison with non-infected animals at day 7, but typically returned to baseline at day 28 (data not shown). No significant differences were identified between the different groups. Similarly, infected rabbits displayed greater weight loss between day 7 and 21 than non-infected rabbits, although none reached the criteria required for early exclusion (data not shown).

Post-euthanasia contact radiographs showed radiographic signs of osteomyelitis in the infected rabbits (Fig. 4; left column), compared to uninfected rabbits (Fig. 4; right column). The osteotomy line was more clearly visible in infected animals, with additional periosteal reaction or osteolysis visible in all cases. The

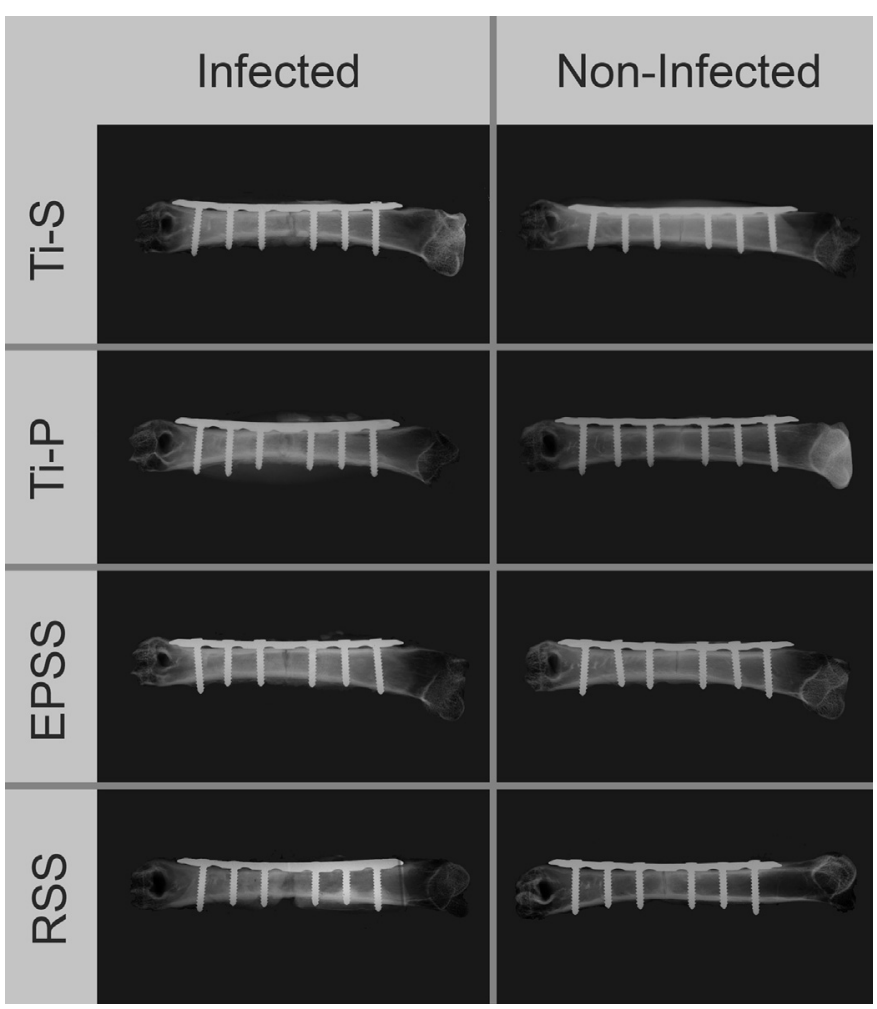

Fig. 4. After the four-week observation period, animals were euthanized and contact radiographs taken. Images including both infected and non-infected examples of each group are shown. All non-infected examples were confirmed to be culture negative. All infected examples were selected on the basis of a positive bacterial yield in the bone. 


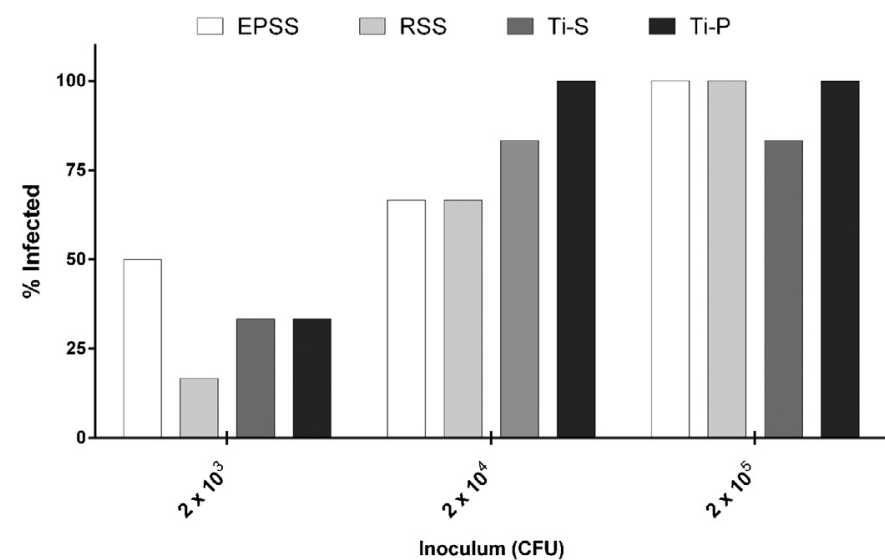

Fig. 5. Implants of each type were placed in $n=6$ rabbits and challenged with three different bacterial doses. The infection rate at each dose is shown.

non-infected animals on the other hand displayed signs of healing, with the osteotomy line less visible indicating the healing process already had begun. There was no marked difference between the implant groups in terms of osteotomy healing as revealed by contact radiograph. One striking (yet expected) feature of the RSS implanted animals was the abundant boney on-growth to the plates in all cases, which was much less observed for each of the remaining groups, and least of all on the EPSS and Ti-P groups.

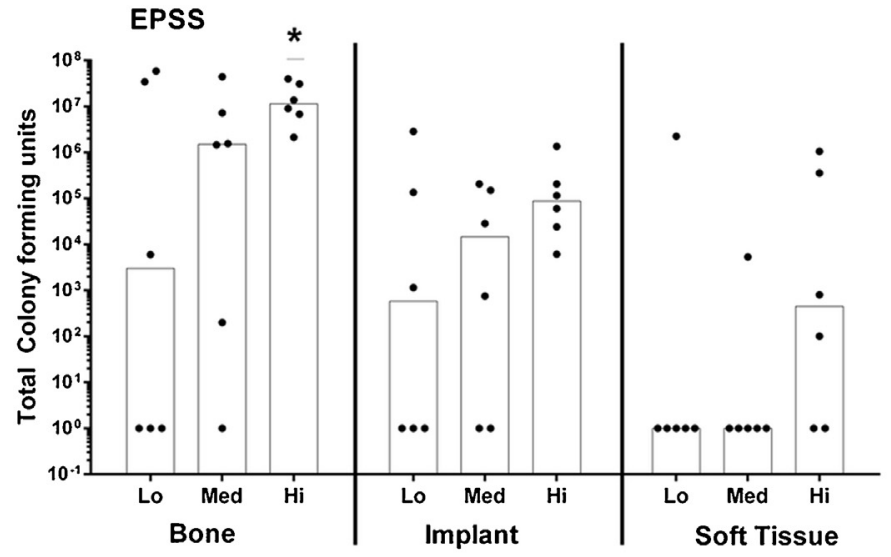

$\mathrm{Ti}-\mathrm{S}$

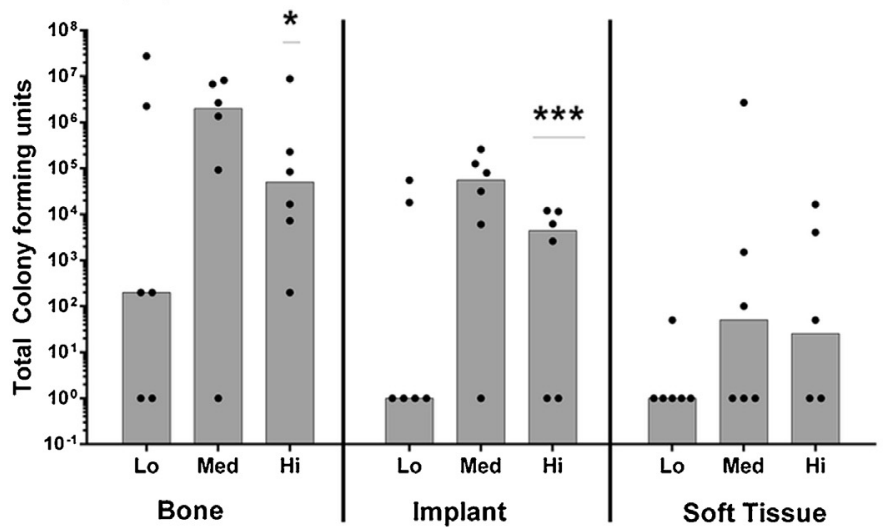

Infection rate

Of the 72 rabbits included in the final analysis, 50 were finally deemed to have developed an infection. On a group-by-group basis, this equated to an overall infection rate of: EPSS 72\%; RSS 61\%; Ti-S 67\%; Ti-P 78\%. The individual infection rate for each group at each inoculum is shown in Fig. 5 . The $\mathrm{ID}_{50}$, which is indicative of the infection susceptibility based on the outcome of each dose was found to be: EPSS $3.89 \times 10^{3} \mathrm{CFU}$; RSS $8.23 \times 10^{3}$ CFU; Ti-S $5.66 \times 10^{3}$ CFU; Ti-P $3.41 \times 10^{3} \mathrm{CFU}$.

\section{Quantitative bacteriology}

The total viable bacterial count from each tissue location is shown in Fig. 6. Overall, bacterial counts increased with increasing inoculum, particularly between the lowest inoculum and the two higher inocula. Furthermore, the bacterial burden was lower in the soft tissue samples overall than in either the bone or implant samples, indicating that the infection model replicates a deep infection and not a superficial wound infection.

\section{Discussion}

Fracture fixation devices are implanted into a growing number of patients each year [19]. With the increase in number of surgical interventions, the number of complications such as implantrelated infection will inevitably rise. All strategies that help to reduce these complications will not only lead to a faster restoration
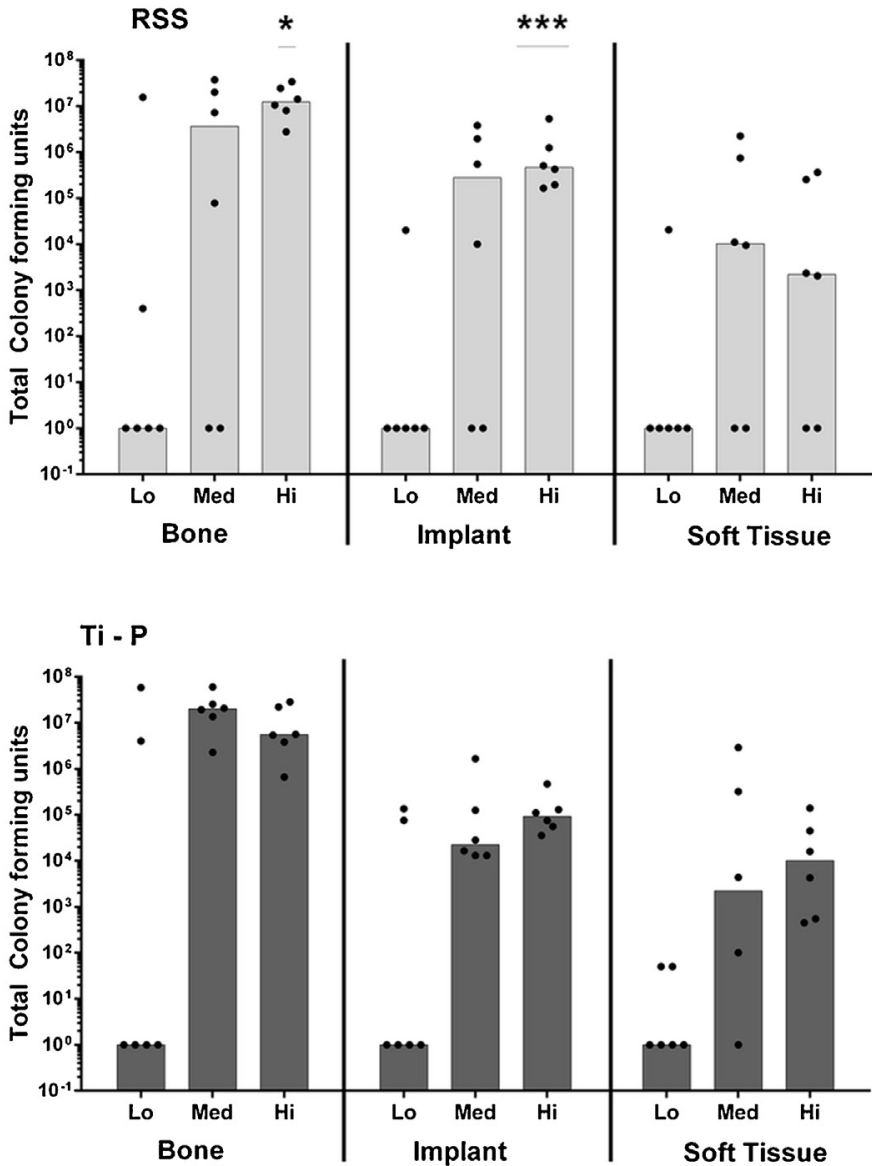

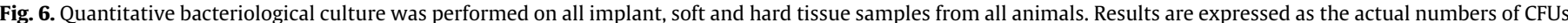

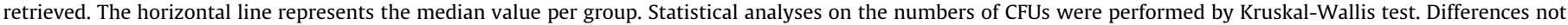
significant unless stated $\left({ }^{*} p<0.005 ;{ }^{* * *} p<0.001\right)$. 
of function, but will also help reduce the resultant healthcare costs. As previously mentioned, the fixation device used and its design have been shown to influence the susceptibility to infection [9]. In the present preclinical model with an osteotomy to represent a fracture, we focussed on the role of implant material (titanium and stainless steel) and surface topography (polished or rough) as influencing factors on the development of implant-related infection.

The results in this study reveal that there is no significant difference in infection risk between the different metal/surface groups based on the $\mathrm{ID}_{50}$ approach in a fracture model. Although some minor differences in $\mathrm{ID}_{50}$ were seen, the magnitude does not indicate any particular material would offer substantial improvements in risk profile over any other. Certainly the magnitude of the reduction in $\mathrm{ID}_{50}$ is below that observed for the change from compression plating to locked plating [9], which remains to date the single greatest material-based factor in reducing infection risk identified in preclinical studies after plating, short of an active antibiotic loaded coating e.g. [20]. One reason that implant design (i.e. DCP versus $\mathrm{LCP}$ ) can reduce infection rates is believed to be due to the fact that infection can spreads along any contiguous area of necrosis, such as may be caused by compressing the plate onto the bone [5]. The introduction of 'biological internal fixation' for example was based on the use of locked internal fixators which protect softtissue vitality and prevent compression necrosis of the bone [21], leading to reduced infection risk.

However, it is important to note that when we compared the bacterial burden (CFU-count) after euthanasia, there were some statistically significant differences between standard titanium (TiS) compared with EPSS and RSS. The magnitude of the difference was quite striking, with an approximate 100-fold reduction in bacterial numbers colonising Ti-S implants in comparison with RSS implants. Earlier experimental data (in vitro and in vivo) have also compared titanium and EPSS with regards to infection susceptibility. In vitro studies show that increasing surface roughness can increase bacterial adhesion, with polished TAN surfaces displaying lower adhesion than standard microrough TAN [6]. Hudetz et al. have shown that titanium offers at most a minor improvement in infection risk in an in vivo model, although the subcutaneous implant does not bear close consideration for fracture care implants [22]. Few clinical studies have compared titanium and EPSS implants in humans. Holzach et al. showed no differences in outcome after the treatment of 256 fractures of the tibia using EPSS or titanium DCPs, both of which had good clinical outcomes [23]. More recently, results from the SPRINT-trial were published [24], wherein the investigators noted that EPSS intramedullary nails had a higher risk of a negative event than titanium equivalents. This observation was attributed to auto-dynamisation in the EPSSgroup, which means that the EPSS locking screws fail during weight bearing, i.e. a mechanical effect. A recent randomised controlled trial comparing the stabilisation of closed femoral shaft fractures in children showed that both titanium and EPSS elastic nails were equally effective treatment modalities, with similar rates of complications [25]. The clinical study with most direct similarity to the preclinical data shown here was performed by Clauss et al. who compared percutaneous EPSS and titanium Kirschner wires used during toe deformity correction [11]. The conclusion of the study was that titanium Kirschner wires had lower bacterial counts and a superior clinical outcome compared to EPSS Kirschner wires, which is indicative that titanium may in fact offer real advantages over steel in terms of bacterial burden, as also revealed in our results. Ultimately, the extent of infection associated with any implant composed of any material, will be a combination of the pathogenic potential of the bacterium, the host response to the infection and the local tissue and vascularity.
Implant materials play a somewhat limited, but important, part in this process.

There may be a number of reasons for the difference in infection burden between the metals. Titanium and stainless steel both offer acceptable mechanical properties and biocompatibility; however, there are certain differences that may contribute to our observation. One explanation for the higher bacterial count in EPSS group may be the fibrous capsule that surrounds these implants [26]. Steel is usually electropolished to a smooth surface, whereas titanium in its standard form has a microrough surface [7]. Tissue adhesion is greater to microrough titanium than to smooth EPSS $[7,27]$ and therefore the fibrous capsule surrounding a liquid-filled void at the interface with EPSS is not observed for microrough surfaces such as Ti-S. The capsule is in effect a dead-space that favours the development of infection $[15,28]$, and this may be a factor in our observation.

With respect to RSS there seems to be another issue. Although roughened steel surfaces lead to quick osseointegration and reduced capsule formation [29], this roughness is also likely to increase initial bacterial adhesion, which is a critical factor in Gristina's "race for the surface" concept [30]. Therefore, in our model (i.e. inoculating the bacteria directly onto the implantation perioperatively) the bacteria win the race and cause an infection, despite the positive effect on osseointegration. It would be interesting to determine if the increased osseointegration offered by RSS implants would offer greater resistance against delayed or late infections, which would be the subject of future investigations.

Another difference between EPSS and Ti-S is that titanium and its alloys form a much thicker oxide layer compared to stainless steel. When the oxide film is mechanically abraded, metal ions may be released from the highly reactive and less biocompatible stainless steel. In general, the most toxic components (cobalt, chromium, nickel and iron) are found in and released from stainless steel [31]. As we know that iron is necessary for the proliferation of bacteria, this may also have an influence on the susceptibility of the implant to infection. When titanium or its alloys have their oxide mechanically damaged, this will instantaneously repair in the presence of oxygen and any ions released are not toxic.

Polishing of titanium and its alloys has been investigated as a potential approach for easing implant removal in fracture care $[26,32,33]$. It has already been clinically implemented. The effect of polishing the surface on infection susceptibility has been evaluated in a non-fracture model [15] and now in this study in a model representing a fracture. Infection susceptibility did not differ significantly between Ti-P and Ti-S implants in our study. Biomechanical testing in the cadaver study, showed no statistical significant differences for failure load and stiffness values between Ti-S and Ti-P LCP, or in screw back-out. This is important information, as it proves surface polishing does not influence biomechanical stability. It was necessary to modify the screw entry procedure for polished screws, which would be an easily overcome manufacturing issue should such screws become clinically available. Overall, these findings support earlier results that surface polishing titanium implants offer eased implant removal, without compromising stability, or infection risk [15,26,32,34].

We are aware of the limitations of this study. First it is difficult to directly extrapolate the results of experimental studies to the clinical setting [35]. Second, as it is an osteotomy model it will not perfectly copy a fracture situation where there may be a diverse fracture pattern many with varying degrees of soft-tissue injury. Thirdly, an important difference with the older studies is that the newer standard titanium implants have a smoother surface (Ra $0.44 \mu \mathrm{m}$ ) compared to the standard titanium plates used in earlier studies (Ra $0.79 \mu$ ), which matched those commercially available at that time. This makes the direct comparison with older studies 
difficult. Finally, although there seems to be a tendency for Ti-S implants to have a lower bacterial burden compared to both EPSS and RSS, it would be difficult to confirm this in a clinical setting.

\section{Conclusion}

In a preclinical in vivo model incorporating fracture biomechanics through an osteotomy, we could not identify any significant differences in susceptibility to infection when comparing titanium and steel implants with conventional (as currently used in the clinics) or modified topographies. The finding that Ti-S has a lower bacterial burden compared to both EPSS and RSS, but only when using a high bacterial inoculum, is interesting and indicates that the material (or its surface) may not influence the infection risk, but rather the infection severity. In theory, the high bacterial load in these animals mimics open fracture cases, where the use of standard titanium implants could be considered a potential benefit. Furthermore, polished titanium implants with potential to reduce complications associated with tissue adherence, are not expected to affect the infection rate, or influence implant stability as shown in this fracture model.

\section{Conflict of interest statement}

The authors declare there are no conflicts of interest relevant to this work.

\section{Acknowledgements}

This work was funded by AOTrauma as part of the Clinical Priority Program Bone Infection. Nora Goudsouzian, Mauro Bluvol, Boyko Gueorguiev, Dieter Wahl, Christoph Sprecher, Benjamin McCarl, Pamela Furlong, all from AO Research Institute Davos, are acknowledged for their expert assistance in the design, performance and discussion of the presented work.

\section{References}

[1] Darouiche RO. Treatment of infections associated with surgical implants. N Engl J Med 2004;350:1422-9.

[2] Schmidt AH, Swiontkowski MF. Pathophysiology of infections after internal fixation of fractures. J Am Acad Orthop Surg 2000:8:285-91.

[3] Moriarty TF, Schlegel U, Perren S, Richards RG. Infection in fracture fixation: can we influence infection rates through implant design? J Mater Sci Mater Med 2010;21:1031-5.

[4] Arens S, Schlegel U, Printzen G, Ziegler WJ, Perren SM, Hansis M. Influence of materials for fixation implants on local infection. An experimental study of steel versus titanium DCP in rabbits. J Bone Joint Surg Br 1996;78:647-51.

[5] Eijer H, Hauke C, Arens S, Printzen G, Schlegel U, Perren SM. PC-Fix and local infection resistance-influence of implant design on postoperative infection development, clinical and experimental results. Injury 2001;32(Suppl 2):B38-43.

[6] Harris LG, Meredith DO, Eschbach L, Richards RG. Staphylococcus aureus adhesion to standard micro-rough and electropolished implant materials. J Mater Sci Mater Med 2007;18:1151-6.

[7] Hayes JS, Richards RG. The use of titanium and stainless steel in fracture fixation. Expert Rev Med Dev 2010;7:843-53.

[8] Ruedi TP, Buckley RE, Moran CG, Ito K, Perren SM, Richards RG, et al. AO principles of fracture management 2. Thieme; 2007.

[9] Schlegel U, Perren SM. Surgical aspects of infection involving osteosynthesis implants: implant design and resistance to local infection. Injury 2006;37(Suppl 2): S67-73.

[10] Melcher GA, Hauke C, Metzdorf A, Perren SM, Printzen G, Schlegel U, et al. Infection after intramedullary nailing: an experimental investigation on rabbits. Injury 1996;27(Suppl 3):SC23-6.
[11] Clauss M, Graf S, Gersbach S, Hintermann B, Ilchmann T, Knupp M. Material and biofilm load of $\mathrm{K}$ wires in toe surgery: titanium versus stainless steel. Clin Orthop Relat Res 2013;471:2312-7.

[12] Soultanis KC, Pyrovolou N, Zahos KA, Karaliotas GI, Lenti A, Liveris I, et al. Late postoperative infection following spinal instrumentation: stainless steel versus titanium implants. J Surg Orthop Adv 2008;17:193-9.

[13] Di Silvestre M, Bakaloudis G, Lolli F, Giacomini S. Late-developing infection following posterior fusion for adolescent idiopathic scoliosis. Eur Spine J 2011:20(Suppl 1):S121-7.

[14] Pieske O, Geleng P, Zaspel J, Piltz S. Titanium alloy pins versus stainless steel pins in external fixation at the wrist: a randomized prospective study. J Trauma 2008;64:1275-80.

[15] Moriarty TF, Debefve L, Boure L, Campoccia D, Schlegel U, Richards RG. Influence of material and microtopography on the development of local infection in vivo: experimental investigation in rabbits. Int J Artif Organs 2009;32:663-70.

[16] Arens D, Wilke M, Calabro L, Hackl S, Zeiter S, Zderic I, et al. A rabbit humerus model of plating and nailing osteosynthesis with and without Staphylococcus aureus osteomyelitis. Eur Cell Mater 2015;30:148-62.

[17] Campoccia D, Montanaro L, Moriarty TF, Richards RG, Ravaioli S, Arciola CR. The selection of appropriate bacterial strains in preclinical evaluation of infection-resistant biomaterials. Int J Artif Organs 2008;31:841-7.

[18] Reed LJ, Muench H. A simple method of estimating fifty percent endpoints. Am J Epidemiol 1938;27:493-7.

[19] Trampuz A, Zimmerli W. Diagnosis and treatment of infections associated with fracture-fixation devices. Injury 2006;37(Suppl 2):S59-66.

[20] Metsemakers WJ, Emanuel N, Cohen O, Reichart M, Potapova I, Schmid T, et al A doxycycline-loaded polymer-lipid encapsulation matrix coating for the prevention of implant-related osteomyelitis due to doxycycline-resistant methicillin-resistant Staphylococcus aureus. J Control Release 2015;209:47-56.

[21] Perren SM. Evolution of the internal fixation of long bone fractures. The scientific basis of biological internal fixation: choosing a new balance between stability and biology. J Bone Joint Surg Br 2002;84:1093-110.

[22] Hudetz D, Ursic Hudetz S, Harris LG, Luginbuhl R, Friederich NF, Landmann R. Weak effect of metal type and ica genes on staphylococcal infection of titanium and stainless steel implants. Clin Microbiol Infect 2008;14:1135-45.

[23] Holzach P, Matter P. The comparison of steel and titanium dynamic compression plates used for internal fixation of 256 fractures of the tibia. Injury 1978; 10:120-3.

[24] Schemitsch EH, Bhandari M, Guyatt G, Sanders DW, Swiontkowski M, Tornetta $\mathrm{P}$, et al. Prognostic factors for predicting outcomes after intramedullary nailing of the tibia. J Bone Joint Surg Am 2012;94:1786-93.

[25] Goyal N, Aggarwal AN, Mishra P, Jain A. Randomized controlled trial comparing stabilization of fresh close femoral shaft fractures in children with titanium elastic nail system versus stainless steel elastic nail system. Acta Orthop Belg 2014;80:69-75.

[26] Hayes JS, Seidenglanz U, Pearce AI, Pearce SG, Archer CW, Richards RG. Surface polishing positively influences ease of plate and screw removal. Eur Cell Mater 2010;19:117-26.

[27] Hayes JS, Richards RG. Surfaces to control tissue adhesion for osteosynthesis with metal implants: in vitro and in vivo studies to bring solutions to the patient. Expert Rev Med Dev 2010;7:131-42.

[28] Harris LG, Richards RG. Staphylococci and implant surfaces: a review. Injury 2006;37(Suppl 2):S3-14.

[29] Klöppel H. Influence of stainless steel implant surface microtopography on bony integration. Vetsuisse-Fakultaet der Universitaet Bern; 2004.

[30] Gristina AG, Hobgood CD, Webb LX, Myrvik QN. Adhesive colonization of biomaterials and antibiotic resistance. Biomaterials 1987:8:423-6.

[31] Krischak GD, Gebhard F, Mohr W, Krivan V, Ignatius A, Beck A, et al. Difference in metallic wear distribution released from commercially pure titanium compared with stainless steel plates. Arch Orthop Trauma Surg 2004;124: 104-13.

[32] Hayes JS, Vos DI, Hahn J, Pearce SG, Richards RG. An in vivo evaluation of surface polishing of TAN intermedullary nails for ease of removal. Eur Cell Mater 2009;18:15-26.

[33] Sinicropi SM, Su BW, Raia FJ, Parisien M, Strauch RJ, Rosenwasser MP. The effects of implant composition on extensor tenosynovitis in a canine distal radius fracture model. J Hand Surg Am 2005;30:300-7.

[34] Moriarty TF, Campoccia D, Nees SK, Boure LP, Richards RG. In vivo evaluation of the effect of intramedullary nail microtopography on the development of local infection in rabbits. Int J Artif Organs 2010;33:667-75.

[35] Moriarty TF, Grainger DW, Richards RG. Challenges in linking preclinical antimicrobial research strategies with clinical outcomes for device-associated infections. Eur Cell Mater 2014;28:112-28. discussion 28. 